\title{
Optical Wireless Data Transfer Through Biotissues: Practical Evidence and Initial Results
}

\author{
Iqrar Ahmed ${ }^{1}$, Alexander Bykov $^{2}$, Alexey Popov $^{2}$, Igor Meglinski $^{2}$, Marcos Katz $^{1}$ \\ ${ }^{1}$ Centre for Wireless Communications \\ ${ }^{2}$ Opto-Electronics and Measurement Techniques \\ University of Oulu, Finland \\ iqrar.ahmed@oulu.fi
}

\begin{abstract}
Light has been used in many medical applications to monitor health status and diagnose diseases. Examples include optical sensing through nearinfrared (NIR) spectroscopy, optical coherence tomography, and pulse oximetry. In this article, we propose and demonstrate digital communications through biological tissues using near-infrared light. There are many possible uses to an optical system transmitting information across tissues. In current practices, implants predominantly use radio frequency (RF) radiation for communication. However, molecular biology restricts use of the RF in terms of power, frequency etc., while interference and security issues represent technological challenges in RF communication. In this paper, we demonstrate a novel way of employing NIR light for wireless transmission of data through biological tissues. A phantom mimicking a biological tissue is illuminated with a NIR $810 \mathrm{~nm}$ wavelength light-emitting diode (LED), and a light detector with line-of-sight alignment is placed on receiving end. An experimental testbed for Optical Communications through Biotissue (OCBT) was designed and implemented using mostly off-the-shelf components. Measurements for different levels of optical output power and thicknesses were carried out. Transmission rates as high as several tens of kilobits-per-second across several millimeters of tissues were achieved. Hardware limitations in modulating the baseband signal prevented achieving higher data rates. In addition, a high-resolution picture was successfully transmitted through biotissue. The communication system as well as details of the testbed implementations are presented in this paper. Moreover, initial performance measures as well as suggestions for potential use of this optical communication system are also presented and discussed.
\end{abstract}

Keywords: Near-infrared Communications, Biological Tissue, Optical Wireless Communications, Implantable Medical Devices, Medical Wireless Communications, Medical Technology, WBAN.

\section{$1 \quad$ Introduction}

In the past decades, there has been an increasing interest in applying wireless communications to the field of medical ICT. Examples include the development of wireless personal and body area networks (WPAN and WBAN, respectively). On-body 
and in-body wireless sensors can be deployed on and in the patient's body to monitor physiological variables and further relay this information to a processing node. Radio technology is predominantly used for transmitting data within hospitals and for personalized healthcare monitoring. Medical implant communication systems (MICS), WBANs and WPANs typically exploit radio to communicate with implants and sensors for patient diagnostics, and treatment [1-4].

In recent years, the types and roles of controllable body implants have significantly increased. IMD, implantable medical device, is the generic name to refer to these electronic implants. Typical applications of IMD include monitoring and treatment of cardiac, neurologic and sensory problems as well as automatic deliver of medication. In general, IMDs can be controlled wirelessly to change settings or operating modes, to activate functionalities, etc. As radio communications is the typical way to communicate with electronic implants, these devices are vulnerable to cyber-attacks (i.e., unauthorized use), as it has been demonstrated recently in a number of planned attacks to demonstrate the real risk of commercial devices are prone to encounter [5-7]. The term "brainjacking" has been recently coined to describe a security breach allowing a remote user to control a brain implant [8].

In this paper, we demonstrate the potential of utilizing light instead of radio to carry out wireless communications to and from IMDs. In particular, we use near infrared (NIR) as at these wavelengths, propagation through biotissues is significantly more favorable compared to the case of visible light. Optical communications have numerous advantages over the RF, such as security and privacy, i.e., it cannot be hacked remotely; safety i.e., there is no radiation exposure to human body; noninterference nature i.e., it does not interfere with RF devices, etc. Light-emitting diodes (LEDs) are inexpensive and can emit a broad range of wavelength spectrum. Moreover, power consumption of optical transceivers could be very low. We show that a NIR LED of $810 \mathrm{~nm}$ can transmit data through a dense layer of biotissues. A line-of- sight (LOS) communication link is established between a LED source and photodiode receiver, where a biotissue works as the optical channel. We successfully transmitted a high-resolution image file through the phantom. For the experiments, optical phantoms as well as real bio tissues (e.g., skull) were used. There are numerous challenges in transmitting data through biotissues. Indeed, biotissues are characterized by very high attenuation due to light absorption, reflection within the dense tissue layers and anisotropic properties of biotissues. The photothermal effect due to illumination of organ is worth mentioning, when biotissues are illuminated for a long time. The photothermal effect can increase tissue temperature [9], thus prevention practices are recommended by ICNIRP [10].

In this paper, we describe an experimental testbed that was implemented as a proof of concept. Practical performance measurements were carried out and results are also reported and discussed here. In general, results suggest that transmission optical information over biotissues is possible. Basic performance measures such as data throughput and range can be enhanced by increasing transmitted optical power, though particular care should be taken with the maximum allowed power density.

This paper is organized as follows. Section 2 presents the system model considered in this paper. In Section 3 the developed testbed is introduced, including its key parts, 
namely the front end for wireless data transmission as well as the biotissue- mimicking phantom. Section 4 presents some performance results of the system, while Section 5 discusses use cases and applications. Discussions are presented in Section 6. Finally, in Section 7 the conclusions of this work are presented.

\section{System Model}

Figure 1 depicts the basic concept considered in this paper. Two cases are described. First, an IMD connected to an external node through optical links. Transmission can be in both directions, namely to-body (e.g., control signal transmitted to the IMD) and from body (information or sensor reading transmitted from the IMD for further analysis). The second case considers intra-body communications, where two in-body devices (IMDs, sensors or nodes of a network) exchange information, for instance. While the first case has more immediate applications as the use of IMD is growing rapidly, it is expected that the second case will become also very relevant, as advancement in in-body communications and the concept of internet of human body further develop. The range of the optical links is expected to be from millimeters to centimeters.

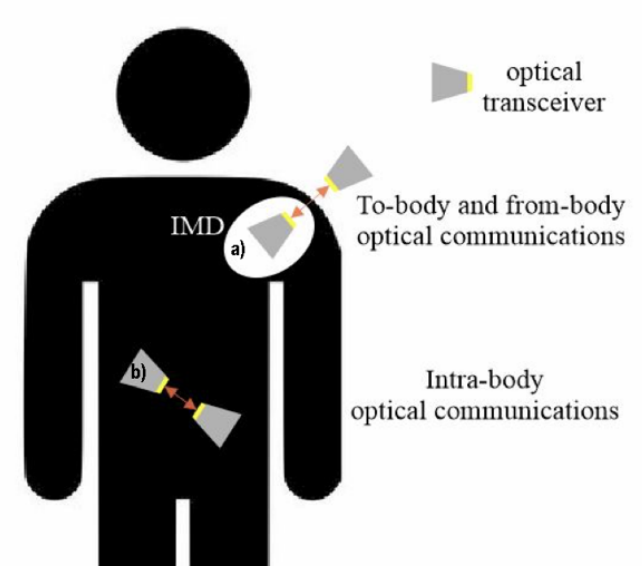

Fig 1. Wireless optical communications: a) to and from an IMD and b) intra-body communications.

\section{A. Optical Communications Link}

The simplified block diagram of the optical transceiver needed to create the communications links is shown in Figure 2. Note that this picture describes a generic twodirectional communication system. Means to control optical power as well as duty 
cycle are also incorporated, as they have a direct impact on the quality and range of the established optical links. Details of the implementation of an experimental testbed are presented in next section. For the purpose of this feasibility study, only a single communication branch was implemented in our testbed.

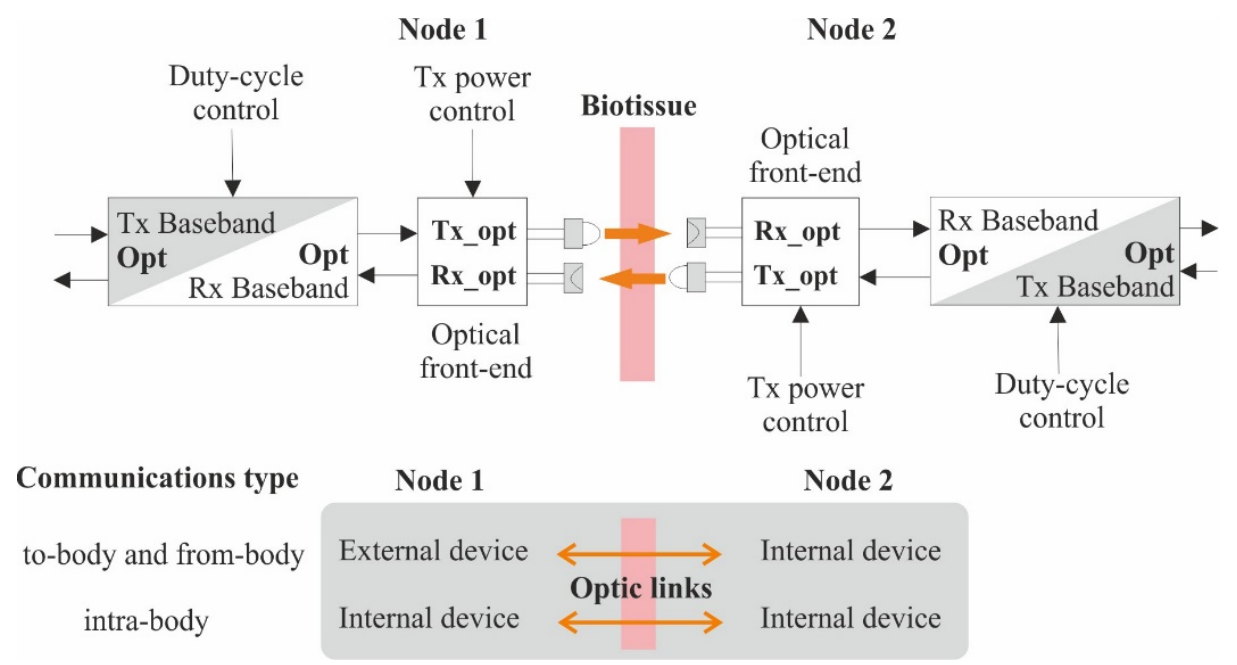

Fig 2. Block diagram of the optical transceiver considered in this paper.

\section{B. Biotissue as an optical media}

Light propagation in a turbid media such as biotissue is challenging. Such medium is characterized by very strong absorption and scattering. Understanding light behavior in biotissues is important due to the multiple applications of light in healthcare. Light, both visible and NIR, is today used for biomedical diagnosis and treatment. Applications include optical tomography, laser surgery, photodynamic therapy. NIR spectroscopy is predominant in transcranial [11] and hemodynamics [12] applications. Numerous non-invasive spectroscopy techniques [13-20] have been studied over the past decades to exploit the harmless nature of light to examine optical properties of biotissue, perform diagnosis as well as to treat malignant tissues. In some cases, therapeutic applications using visible and infrared light have been demonstrated to alleviate pathological disorders, wound healing, pain, and inflammation [21-22]. These therapeutic applications either utilize low-level light, termed as low-level light therapy (LLLT) or non-ionizing light sources termed as photobiomodulation (PBM). The aim of using low level light is to stimulate photochemical and photophysical effects in biotissues for remedy and to alleviate the photothermic response from biotissues $[21,23,24]$.

Light interaction with biotissues has been extensively studied to reveal tissue properties such as absorption, scattering, anisotropy [25-27] and further used in modelling. The useful optical or therapeutic window lies between $700 \mathrm{~nm}$ to $1100 \mathrm{~nm}$, where the NIR light faces minimal absorption and scattering, hence maximum penetration in 
biotissues. Scattering, the dominant phenomenon in biotissues, causes light dispersion, reducing rapidly energy density as light propagates. The initial step in utilizing NIR light is to understand the relationship of wavelength and its interaction with biotissues. Next, to study this relationship we utilize a phantom mimicking the biotissue in our experimentations. The characteristics of the phantom must match the optical characteristics of the tissue it is mimicking. Nowadays, these phantoms are fabricated utilizing different biopolymers mixed with numerous dyes and particles [28,29]. As light interact with biotissues, particular care is needed to avoid any possible damage to the tissue. Light energy- and power-density need to be below certain limits to avoid harmful effects [30].

In this paper, we propose and demonstrate the use of biotissues as optical channels for transmitting information. Communication across biotissues has numerous applications as use cases, as discussed later in this paper.

\section{Testbed Implementation}

In this section, we briefly introduce our testbed, the front-end components we utilized for transmitting and receiving data. Next, we discuss the modulation scheme we implemented for modulating the baseband carrier. Finally, we describe the phantom we utilized in our testing.

\subsection{System Overview}

We developed our testbed utilizing mostly off-the-shelf electronics. We utilized USRP modules (Universal Software Radio Peripheral) to implement the receiver and transmitter. GNU radio software was used and we implemented the GNU radio tunnel example [31]. The software is available freely and does not need any licensing to implement. The GNU radio tunnel example creates a tunnel between source and receiving nodes, the software comprises several blocks connected to each other. Among several modulation options available in GNU radio, we chose and implemented Gaussian minimum-shift keying (GMSK) modulation. GMSK is a constant envelope scheme that performs better than other modulation schemes e.g., quadrature phaseshift keying (QPSK) etc., in the optical communications. The slowness of LED to follow the abrupt phase changes in the externally modulated optical signal deteriorates the performance of QPSK [31]. 


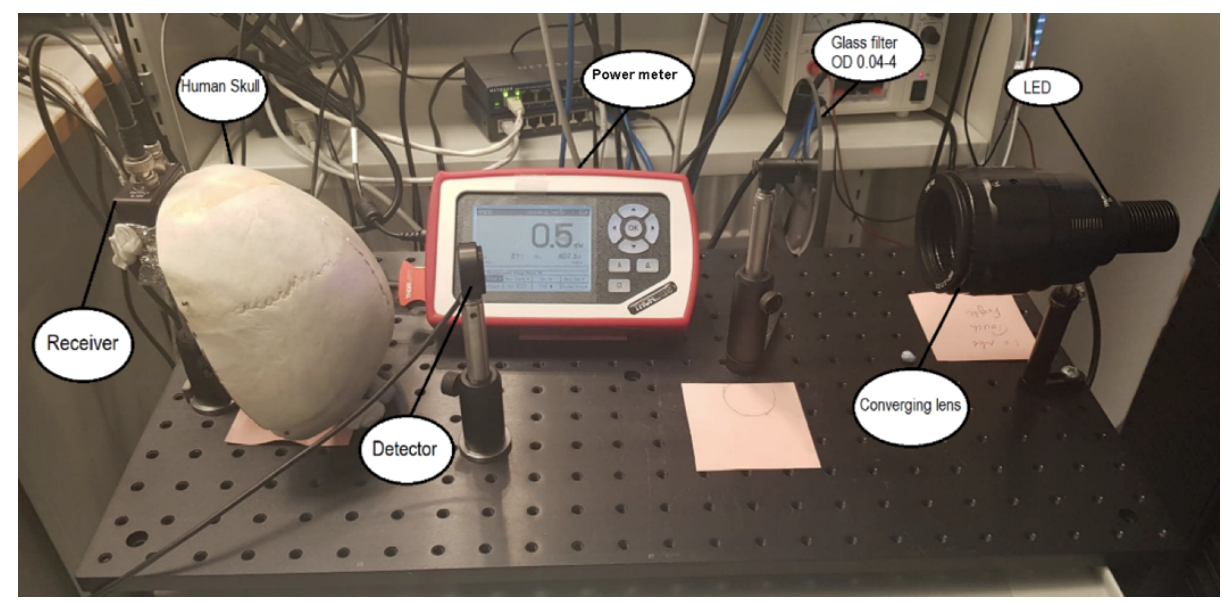

Fig 3. Overview of the testbed, including optical transmitter (right), optical receiver (left) and tissue (skull).

Figure 3 shows the testbed components; the transmitting end on the right transmits data-modulated light (NIR) and the receiving end on the left side receives and further process the optical signal. An optical phantom or real tissue is placed between transmitter and receiver.

\subsection{Transmitter Characteristics}

We have used Thorlabs $810 \mathrm{~nm}$ mounted IR LED [32], the LED has a maximum output power of $325 \mathrm{~mW}$ and has a radiation spot of $1 \mathrm{~mm}^{2}$. The maximum supplied current to the LED is $500 \mathrm{~mA}$. To modulate the LED input current, we have used the Thorlabs DC2200 LED driver [33]. The LED driver comprises a single channel and can supply the LED with as high as 10 A current providing the input voltage to the driver of $50 \mathrm{~V}$. The LED driver also supports external modulation, termed as Small Signal Bandwidth by Thorlabs. The driver can handle sine wave and has a limitation of handling baseband modulated signal within the range from DC-250 kHz. The external modulated signal from USRP is fed to DC bias-Tee that can operate between $100 \mathrm{kHz}$ to $4200 \mathrm{MHz}$ at 0 to $5 \mathrm{~V}$. The bias-Tee adds the DC voltage to RF signal from USRP. Output of bias-Tee is then fed to DC2200 LED driver, the driver feeds the equivalent current to LED. The input current to LED is converted by driver based on modulation coefficient formulae by Thorlabs. The specification sheet of DC 2200 tells that per $1 \mathrm{~V}$, the driver feeds $400 \mathrm{~mA}$ current to LED. The LED can handle between 0 to $3.6 \mathrm{~V}$ forward voltage, so we maintained the DC bias to $3.6 \mathrm{~V}$ when illuminating the LED with full scale. The input to LED can be controlled by changing the applied voltage to bias-Tee. The LED brightness is directly proportional to the percentage of current fed by LED driver. 
The beam collimation is performed by Thorlabs SM2F32B anti-reflective (AR) lens [34]. The lens with adjustable collimation can operate within the range of 650$1050 \mathrm{~nm}$ and the reflective coating is used to alleviate reflection.

\subsection{Receiver Characteristics}

The receiver of our testbed comprises silicon avalanche photodetector APD120A from Thorlabs [35]. The operating range for this detector is from 400-1000 nm. The sensitivity of the detector peaks at $800 \mathrm{~nm}$, the active area of detector is $1 \mathrm{~mm}^{2}$ while it can be increased by attaching a lens to the detector. The output of the receiver is connected to USRP. The signal from the receiver is then demodulated on receiver's end for further processing.

\subsection{Biotissue-mimicking Phantom}

Rigorous experimentation needs to be carried out when measuring optical properties of biotissues. The properties of biotissues degrade over time, thus use of stable biotissue-mimicking phantoms with present properties are of high importance. The phantoms are fabricated in our laboratories using polyvinyl chloride-plastisol (PVCP) and zinc oxide $(\mathrm{ZnO})$ nanoparticles, the properties of the phantom remain the same at least over four months [28,29]. The optical properties i.e., absorption coefficient $\mu a$, scattering coefficient $\mu s$, scattering anisotropy $g$, thickness $L$ and refractive index $n$ of the phantom are tuned to match the properties of the biotissue it mimics.

Transmittance, reflectance, and collimated transmittance of the fabricated phantom are measured and the mentioned optical properties are reconstructed by inverse adding-doubling (IAD) method. The phantoms we used in our experiments mimic skin. We added multiple layers of phantoms to mimic the complex structure of skin.

\section{System Evaluation}

As discussed in section 3.2, a Thorlabs NIR LED was employed, where the LED's input current was modulated through an LED driver DC2200. The output/brightness of the LED is proportional to the input current supplied. When an externally modulated voltage signal is applied to the LED driver, it converts the voltage to an input current for the LED based on modulation coefficient. $1 \mathrm{~V}$ is converted into $400 \mathrm{~mA}$ current. The maximum output of the LED is $325 \mathrm{~mW}$ generated at $500 \mathrm{~mA}$ when an externally modulated voltage of $3.6 \mathrm{~V}$ is applied to LED driver. The output of the LED can be reduced by varying the modulating signal, to avoid damaging the biotissue when exposed for longer durations, due to the photothermal effect. ANSI.Z136.12007 [36] standard on laser safety states that for $830 \mathrm{~nm}$ wavelength an exposure for 1 sec that can generate $2 \mathrm{~W} / \mathrm{cm}^{2}$ power is safe. We measured the optical power applied to the phantom at varying input levels. Table 1 gives an overview of the measured optical power at the phantom at varying input current, the values are well within the safe limit. 
Table 1. The amount of optical power delivered to the phantom.

\begin{tabular}{cc}
\hline LED Input Current $(\mathrm{mA})$ & Optical Power to Phantom $\left(\mathrm{mW} / \mathrm{cm}^{2}\right)$ \\
\hline 10 & 5.3 \\
50 & 22.15 \\
100 & 49.5 \\
200 & 102.8 \\
300 & 147 \\
400 & 195.4 \\
500 & 239.1 \\
\hline
\end{tabular}

We utilized Thorlabs NDC-100C-4 Unmounted Round Variable Density Filter (Uncoated). This linear attenuation filter has $100 \mathrm{~mm}$ in diameter and the optical density (OD) of the filter ranges between $0.04-4$. The filter is utilized to investigate the optical communication at varying OD, as OD corresponds to tissue thickness. Figure 4 shows the amount of power transmitted through filter at different OD, measured at the receiver. These power values are well below the safe limit set by ANSI. Transmitted signal cannot go through for values of OD larger than 3. The top horizontal axis in red represents the equivalent depth of bloodless dermis.

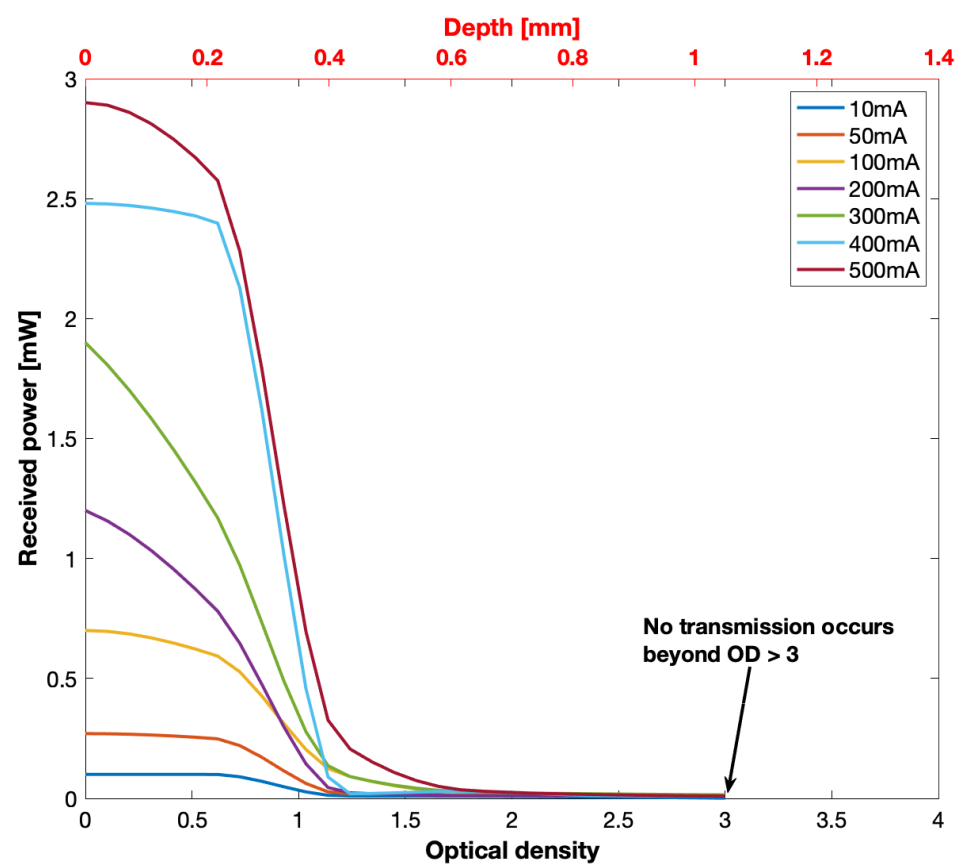

Fig 4. Transmitted power through filter at different optical densities. The equivalent depth for bloodless dermis presented in top (red) horizontal axis.

Figure 5 shows the relationship between thickness and OD for white matter, gery matter, bloodless dermis, muscles and breast. OD is expressed in term of exponential 
decrease in optical intensity when transmitted through biotissues, for OD equal to 1, the incident optical intensity will be decreased 2.71 . For grey matter, $0.2 \mathrm{~mm}$ thickness will decrease incident intensity to 2.71 times, similarly $0.6 \mathrm{~mm}$ slab will provide the OD equivalent to 2 . We estimated the light attenuation for 5 different types of media from which light can pass through. Figure 6 shows that increasing LED output will result in light passing through an increased thickness, this is advantageous when transmitting data with high energy pulses of short duty cycle, in this way data can be transmitted through thicker slabs of phantoms while keeping operating safety below the obligatory limits.

In the experiments, a data throughput of several tens of Kbps was attained. The current testbed set some practical limitations on the maximum achievable data throughput. In our future work, we plan to experiment with more advanced hardware systems. We expect a considerable increase in data rate support.

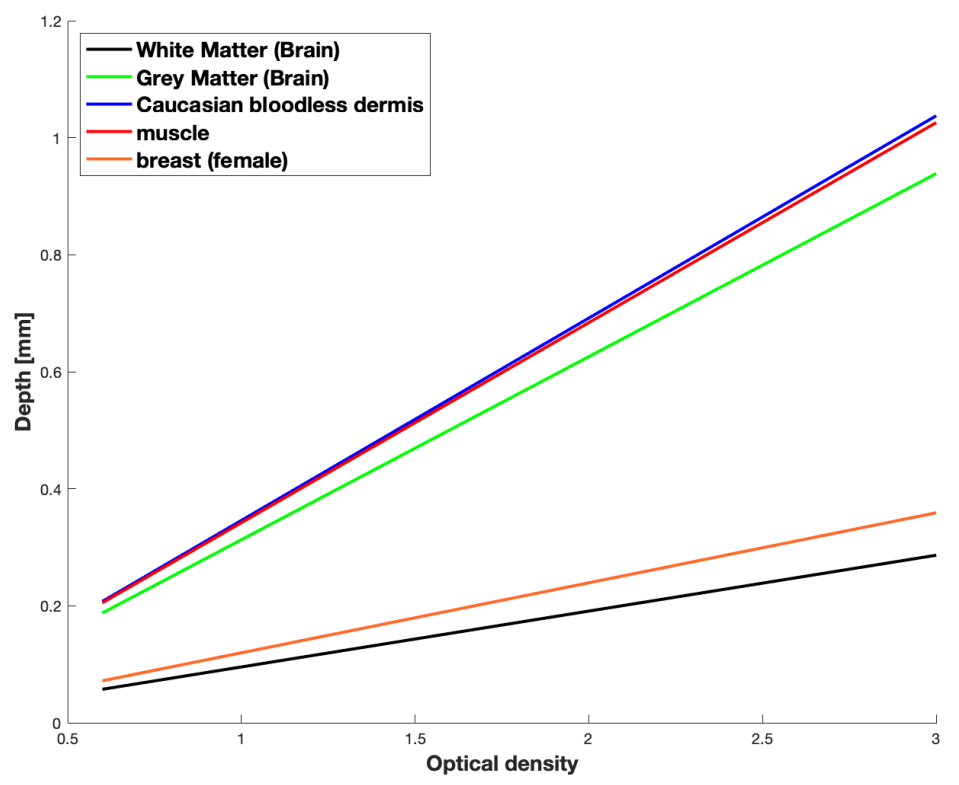

Fig 5. Penetration depth (thickness) of different types of biotissue as a function of optical density. 


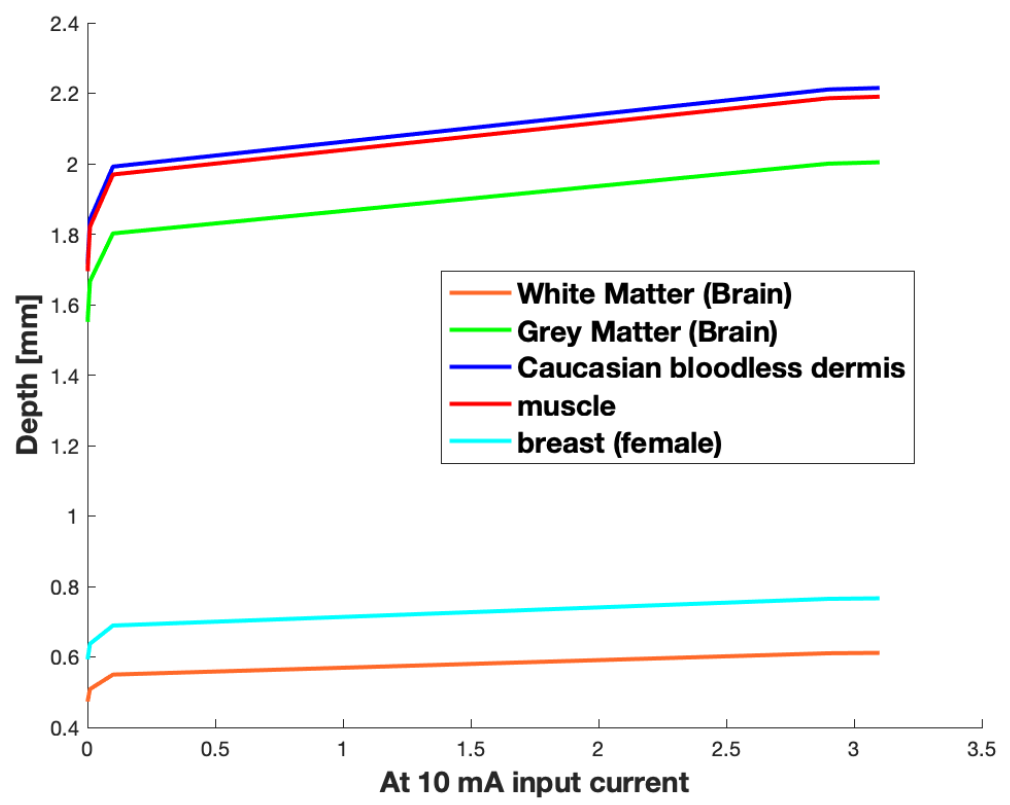

Fig 6. Relationship between the achieveable tissue depth and LED input current.

\section{$5 \quad$ Use Cases and Applications}

Optical Communications through Biotissue (OCBT) has numerous use cases and applications. As discussed, the most immediate application is providing wireless connectivity to IMD. Representative IMDs include cardiac devices (pacemakers and implantable cardioverter defibrillators), neurostimulators (e.g., deep brain stimulators, gastric electrical stimulators, spinal cord stimulators, brain implants, etc.) and medical delivery pumps (e.g., delivering medicines, fluids, nutrients, etc.) Over the years, the types and applications of IMD have increased rapidly.

OCBT offers a number of benefits over RF communications, as summarized next

Security and privacy are some of the most relevant advantages of OCBT; eavesdropping, jamming the communication link or accessing illegally the IMD is extremely difficult as accessing an IMD needs to be done locally, in some cases requiring proximity of the order of centimeters.

Safety: radio exposure is not an issue when using OCTB. However, biotissues and organs could also be damaged by light if high-power light sources are used. Low power light and pulse-modulated carriers are in general preferred for OCBT.

Interference: Radio-based links are prone to be interfered by other radio systems. This is particularly true in uncontrolled environments, e.g., home, where other radio 
systems could be located in the vicinity. In controlled environments, e.g., hospitals, radio links could eventually create interference to highly sensitive equipment. OCBT does not suffer from these limitations.

Energy: Power requirements of optical transceivers can be very low, and this is an important advantage particularly for energy-limited IMD. Furthermore, in some applications where range is very short, e.g., subcutaneous IMD, the same light used to transmit information can be used to power up the complete IMD or some of its functionalities. The possibility of energy harvesting is a unique advantage of OCBT.

Integration to other light-based applications: In principle, this approach can be combined with already well-established light-based healthcare applications. For instance, light from node A to node B could be used to make a diagnosis of the tissue between these nodes. Node B, after making the diagnosis, transmits these results digitally to node A.

Hybrid networks: In order to enhance performance and have a much more flexible connectivity solution (e.g., increase reliability, data rate) OCBT can be combined radio-based transmissions [31, 37].

In-body connectivity: In addition to providing connectivity to IMDs, OCBT can be used to create in-body networks, from simple point-to-point wireless connections to more sophisticated networks involving sensing nodes, gateways, repeaters, and other processing units. In principle in-body nodes as well as IMDs can be nodes of a WBAN.

\section{Discussions}

In this section, we further discuss the results and prospects for the concepts proposed in this paper. We highlight the fact that the above results are exploratory, and additional measurements will be carried out to better characterize the suitability of biotissues as optical media for data transmission. However, results so far are encouraging, and we can already say that even with low optical power, low data rate information can be transmitted through biotissues across some reasonable distances (e.g., centimeters). Using higher optical power and pulsed communications is expected to increase data throughput and range.

There are some limitations associated with the current testbed, which are mostly due to hardware. The use of commercial off-the-shelf components (Thorlabs and National Instruments) limits the achievable data transmission rate in our initial experiments. However, this low data rate is enough for transmitting packets of data as those required by some IMDs. Some IMD applications might require substantially higher data rates. For that, in addition to increasing transmitted power, one can use more advanced modulation schemes. In our experiments, we successfully transmitted a 14 MB of high-resolution image through the biotissue-mimicking phantom.

Since OCBT is a novel technique to transmit data through biotissues, several technological challenges need to be tackled. Moreover, extensive experimentation on phantoms needs to be carried out to further understand this medium. Performance of 
the optical link can be enhanced by exploiting different techniques. Next, some of these techniques are discussed to improve energy efficiency, reliability and range.

Energy efficiency. The OCBT sensors up-fronts i.e., receiver and transmitter, can be configured to work in an interactive mode keeping the up-front in sleep mode for conserving energy. In the battery-operated OCBT sensors, the power consumption needs to be kept as low as possible. The size of the up-front circuitry should be kept small and interactive communication would help to reduce power drainage. The overall size of the sensor and electronic circuitry is important; the package should be kept small to preserve energy for prolonged battery life.

High reliability/integrity. The reliability of data is of utmost importance; possible errors due to partial data reception need be checked and corrected. Reliability can be addressed in many ways, and this needs to be further investigated in this scenario. Increasing transmission power is not a straightforward solution as there are limits on the maximum energy that be radiated into biotissue. The use of diversity is a promising approach, and diversity can be exploited in multiple domains. The sensitivity of the detector can be increased to handle lower power.

Range: In some cases, such as in-body communications or when IMD are deeply implanted, the communications range might become an issue. Increasing transmission power to increase range is not a straightforward solution, as the transmission must comply with regulations limiting the transmitted optical power.

\section{Conclusions}

In this paper, we proposed and demonstrated the use of light to transmit digital information across biotissues. A near infrared LED was used as a light source, as propagation of NIR light in biotissues is more favorable than at other wavelengths. An experimental testbed was implemented, consisting of an optical transmitter, a phantom/biotissue and an optical receiver. The demonstrated Optical Communication through Biotissue (OCBT) technique offers potential benefits over radio, because, among others, the optical communication is secure and safe compared to radio. We discussed the architecture of OCBT used in this study. The data signal modulating the IR LED is controlled through a LED driver. We utilized GMSK modulation in our measurements. In our testbed, proven and inexpensive technology was used. Bidirectional communicational is also possible and it depends on sensor package.

Measurements show that it is feasible to use light to securely transmit information across biotissues. Initial results show that tens of Kbps can be easily achieved in a range of several millimeters. This is enough for many applications with IMD, as they are located well within the demonstrated range. The range and data throughput can be increased by using more advanced modulation schemes. This will be further investigated in the future. In addition, we plan to study the use of pulsed transmission to increase the range of the transmission. Extensive experimentation with different phantoms/tissues will also be carried out to characterize the transmission medium in more detail. 
Several challenges need to be overcome in OCBT to create a truly practical system. Building and embedding the proposed communication system into a small package using system-on-chip (SoC) or other integrated circuit technology is required in order to have a practically usable system. Miniaturization of the optical transceiver is quite feasible, as the communication system is quite simple, for low-rate systems operating over several millimeters. The potential benefits of security and user safety in OCBT make the demonstrated system promising for future medical applications.

\section{References}

1. Bradley, P.D., 2006, November. An ultra low power, high performance medical implant communication system (MICS) transceiver for implantable devices. In 2006 IEEE Biomedical Circuits and Systems Conference (pp. 158-161). IEEE.

2. Chow, E.Y., Morris, M.M. and Irazoqui, P.P., 2013. Implantable RF medical devices: The benefits of high-speed communication and much greater communication distances in biomedical applications. IEEE Microwave Magazine, 14(4), pp.64-73.

3. Chen, Z.N., Liu, G.C. and See, T.S., 2009. Transmission of RF signals between MICS loop antennas in free space and implanted in the human head. IEEE Transactions on Antennas and Propagation, 57(6), pp.1850-1854.

4. Karvonen, H., Mikhaylov, K., Hämäläinen, M., Iinatti, J., Pomalaza-Ráez, C.: Interference of wireless technologies on BLE based WBANs in hospital scenarios. In: Proceedings of the 2017 IEEE 28th Annual International Symposium on Personal, Indoor, and Mobile Radio Communications (PIMRC), 1-6, IEEE, Montreal, Canada (2017).

5. Carmen Camara, Pedro Peris-Lopez and Juan E.Tapiadora, Security and privacy issues in implantable medical devices: A comprehensive survey, Journal of Biomedical Informatics, Volume 55, June 2015, Pages 272-289.

6. Jake L Beavers, Michael Faulks and Jims Marchang, Hacking NHS Pacemakers: A Feasibility Study, 2019 IEEE 12th International Conference on Global Security, Safety and Sustainability (ICGS3), 16-18 Jan. 2019, London, UK.

7. Aliya Tabasum, Zeineb Safi, Wadha AlKhater and Abdullatif Shikfa, Cybersecurity Issues in Implanted Medical Devices, 2018 International Conference on Computer and Applications (ICCA), 2018.

8. Laurie Pycroft, Sandra G.Boccard, Sarah L.F.Owen, John F.Stein, James J.Fitzgerald, Alexander L.Green and Tipu Z. Aziz, Brainjacking: Implant Security Issues in Invasive Neuromodulation, World Neurosurgery, Volume 92, August 2016, Pages 454-462

9. Bozkurt, A. and Onaral, B., 2004. Safety assessment of near infrared light emitting diodes for diffuse optical measurements. biomedical engineering online, 3(1), p.9.

10. International Commission on Non-Ionizing Radiation Protection, 2013. ICNIRP guidelines on limits of exposure to incoherent visible and infrared radiation. Health Physics, 105(1), pp.74-96.

11. Jagdeo, J.R., Adams, L.E., Brody, N.I. and Siegel, D.M., 2012. Transcranial red and near infrared light transmission in a cadaveric model. PloS one, 7(10), p.e47460.

12. Zhang, H., Salo, D.C., Kim, D.M., Komarov, S., Tai, Y.C. and Berezin, M.Y., 2016. Penetration depth of photons in biological tissues from hyperspectral imaging in shortwave infrared in transmission and reflection geometries. Journal of biomedical optics, 21(12), p.126006. 
13. Mil'Shtein, S., 2006. Infrared scanning for biomedical applications. Scanning, 28(5), pp.274-277.

14. Myllylä, T., Harju, M., Korhonen, V., Bykov, A., Kiviniemi, V. and Meglinski, I., 2018. Assessment of the dynamics of human glymphatic system by near-infrared spectroscopy. Journal of biophotonics, 11(8), p.e201700123.

15. Korhonen, V.O., Myllylä, T.S., Kirillin, M.Y., Popov, A.P., Bykov, A.V., Gorshkov, A.V., Sergeeva, E.A., Kinnunen, M. and Kiviniemi, V., 2014. Light propagation in NIR spectroscopy of the human brain. IEEE Journal of Selected Topics in Quantum Electronics, 20(2), pp.289-298.

16. Alarousu, E., Hast, J.T., Kinnunen, M.T., Kirillin, M.Y., Myllyla, R.A., Plucinski, J., Popov, A.P., Priezzhev, A.V., Prykari, T., Saarela, J. and Zhao, Z., 2004, August. Noninvasive glucose sensing in scattering media using OCT, PAS, and TOF techniques. In Saratov Fall Meeting 2003: Optical Technologies in Biophysics and Medicine V (Vol. 5474, pp. 33-42). International Society for Optics and Photonics.

17. Bykov, A., Hautala, T., Kinnunen, M., Popov, A., Karhula, S., Saarakkala, S., Nieminen, M.T., Tuchin, V. and Meglinski, I., 2016. Imaging of subchondral bone by optical coherence tomography upon optical clearing of articular cartilage. Journal of biophotonics, 9(3), pp.270-275.

18. Popov, A.P., Karmenyan, A.V., Bykov, A.V., Khaydukov, E.V., Nechaev, A.V., Bibikova, O.A., Panchenko, V.Y., Semchishen, V.A., Akhmanov, A.S., Sokolov, V.I. and Kinnunen, M.T., 2013, May. High-resolution deep-tissue optical imaging using anti-Stokes phosphors. In European Conference on Biomedical Optics (p. 88010C). Optical Society of America.

19. Meglinsky, I.V. and Matcher, S.J., 2001. Modelling the sampling volume for skin blood oxygenation measurements. Medical and Biological Engineering and Computing, 39(1), pp.44-50.

20. Bonesi, M., Proskurin, S.G. and Meglinski, I.V., 2010. Imaging of subcutaneous blood vessels and flow velocity profiles by optical coherence tomography. Laser Physics, 20(4), pp.891-899.

21. Tsai, S.R. and Hamblin, M.R., 2017. Biological effects and medical applications of infrared radiation. Journal of Photochemistry and Photobiology B: Biology, 170, pp.197-207.

22. Henderson, T.A. and Morries, L.D., 2015. Near-infrared photonic energy penetration: can infrared phototherapy effectively reach the human brain?. Neuropsychiatric disease and treatment, 11, p.2191.

23. de Freitas, L.F. and Hamblin, M.R., 2016. Proposed mechanisms of photobiomodulation or low-level light therapy. IEEE Journal of selected topics in quantum electronics, 22(3), pp.348-364.

24. Anders, J.J., Lanzafame, R.J. and Arany, P.R., 2015. Low-level light/laser therapy versus photobiomodulation therapy.

25. Kim, N.J. and Lim, H.S., 1998, October. Measurements of absorption coefficients within biological tissue in vitro. In Proceedings of the 20th Annual International Conference of the IEEE Engineering in Medicine and Biology Society. Vol. 20 Biomedical Engineering Towards the Year 2000 and Beyond (Cat. No. 98CH36286) (Vol. 6, pp. 2960-2962). IEEE.

26. Ntziachristos, V., Ripoll, J. and Weissleder, R., 2002. Would near-infrared fluorescence signals propagate through large human organs for clinical studies?. Optics letters, 27(5), pp.333-335.

27. Jacques, S.L., 2013. Optical properties of biological tissues: a review. Physics in Medicine \& Biology, 58(11), p.R37. 
28. Wróbel, M.S., Popov, A.P., Bykov, A.V., Kinnunen, M., Jędrzejewska-Szczerska, M. and Tuchin, V.V., 2015. Multi-layered tissue head phantoms for noninvasive optical diagnostics. Journal of Innovative Optical Health Sciences, 8(03), p.1541005.

29. Wróbel, M.S., Popov, A.P., Bykov, A.V., Kinnunen, M., Jędrzejewska-Szczerska, M. and Tuchin, V.V., 2015. Measurements of fundamental properties of homogeneous tissue phantoms. Journal of biomedical optics, 20(4), p.045004.

30. International Commission on Non-Ionizing Radiation Protection. (2000). ICNIRP statement on light-emitting diodes (LEDs) and laser diodes: implications for hazard assessment. Health Physics, 78(6), 744-752.

31. Saud, M.S., Ahmed, I., Kumpuniemi, T. and Katz, M., 2019. Reconfigurable optical-radio wireless networks: Meeting the most stringent requirements of future communication systems. Transactions on Emerging Telecommunications Technologies, 30(2), p.e3562.

32. https://www.thorlabs.com/drawings/b1ef4257936da4d4-4B402694-0F84-14D672D3E2437F4E3C75/M810L3-SpecSheet.pdf

33. https://www.thorlabs.com/drawings/b1ef4257936da4d4-4B402694-0F84-14D672D3E2437F4E3C75/DC2200-Manual.pdf

34. https://www.thorlabs.com/thorproduct.cfm?partnumber=SM2F32-B

35. https://www.thorlabs.com/drawings/b1 ef4257936da4d4-4B402694-0F84-14D672D3E2437F4E3C75/APD120A M-Manual.pdf

36. https://assets.lia.org/s3fs-public/pdf/ansi-standards/samples/ANSI\%20Z136.1_sample.pdf

37. Ahmed, I., Kumpuniemi, T. and Katz, M., (2018). A Hybrid Optical-Radio Wireless Network Concept for the Hospital of the Future, BodyNets, Oulu.

\section{Acknowledgement}

This research has been funded by Academy of Finland HERONET project and partially funded by Academy of Finland (6Genesis Flagship - grant 318927 and grants 290596, 314369). 